\title{
A compactness result of Janson type for bilinear operators
}

\author{
Fernando Cobos ${ }^{1, *}$ \\ Departamento de Análisis Matemático y Matemática Aplicada, Facultad de Matemáticas, \\ Universidad Complutense de Madrid, Plaza de Ciencias 3, 28040 Madrid. Spain. \\ Luz M. Fernández-Cabrera ${ }^{1}$ \\ Sección Departamental del Departamento de Análisis Matemático y Matemática Aplicada, \\ Facultad de Estudios Estadísticos, Universidad Complutense de Madrid, 28040 Madrid, \\ Spain. \\ Antón Martínez ${ }^{1}$ \\ Departamento de Matemática Aplicada I, Escuela de Ingeniería Industrial, Universidad de \\ Vigo, 36200 Vigo, Spain.
}

\begin{abstract}
We establish a compactness interpolation result for bilinear operators of the type proved by Janson for bounded bilinear operators. We also give an application to compactness of convolution operators.
\end{abstract}

Keywords: Compact bilinear operators; real interpolation; Lorentz spaces; convolution operators.

2010 MSC: Primary 46M35, 46B70, 47H60. Secondary 46E30.

\section{Introduction}

Bilinear (and multilinear) interpolation results for the real method [24] and for the complex method [6] have found interesting applications in analysis and operator theory. The results for the real method are not so satisfactory as for the complex method. Namely, if $\left(A_{0}, A_{1}\right),\left(B_{0}, B_{1}\right),\left(E_{0}, E_{1}\right)$ are Banach couples and the bilinear operator $T$ satisfies that $T: A_{j} \times B_{j} \longrightarrow E_{j}$ is bounded, $j=0,1$, then the complex bilinear interpolation theorem yields that

$$
T:\left[A_{0}, A_{1}\right]_{\theta} \times\left[B_{0}, B_{1}\right]_{\theta} \longrightarrow\left[E_{0}, E_{1}\right]_{\theta} \quad \text { boundedly }
$$

for any $0<\theta<1$. The corresponding result for the real method reads that

$$
T:\left(A_{0}, A_{1}\right)_{\theta, q_{1}} \times\left(B_{0}, B_{1}\right)_{\theta, q_{2}} \longrightarrow\left(E_{0}, E_{1}\right)_{\theta, q} \text { boundedly }
$$

\footnotetext{
*Corresponding author.

Email addresses: cobos@mat.ucm.es (Fernando Cobos ), luz_fernandez-c@mat.ucm.es (Luz M. Fernández-Cabrera), antonmar@uvigo.es (Antón Martínez )

${ }^{1}$ The authors have been supported in part by MTM2017-84058-P (AEI/FEDER, UE).
} 
provided that $0<\theta<1,1 \leq q_{1}, q_{2}, q \leq \infty$ and

$$
1 / q \leq 1 / q_{1}+1 / q_{2}-1 .
$$

See the papers by Calderón [6] and Lions and Peetre [24] or the books by Bergh and Löfström [4, Theorem 4.4.1 and Exercise 3.13.5] and Triebel [31, Section 1.19.5]. The result for the real method is also valid for couples of quasiBanach spaces (see the papers by Karadzhov [20] and König [21]).

However, working with convolution operators among Lorentz spaces, it was shown by O'Neil [26] that condition (1.1) can be improved to

$$
1 / q \leq 1 / q_{1}+1 / q_{2} .
$$

An abstract result in this direction was established by Janson [19] with the effect that under additional information on the bilinear operator $T$ (which is the case of convolution operators) then the bilinear interpolation theorem for the real method holds if (1.2) is satisfied. Janson's theorem works not only for Banach couples but also for quasi-Banach couples.

Compactness is another important property that a bilinear operator may have. Recently, in the papers by Bényi and Torres [3], Bényi and Oh [2], Hu [17] and other authors, it has been shown that this kind of operators arise rather naturally in harmonic analysis. This fact has been a motivation for the research on the interpolation properties of compact bilinear operators. In fact, Calderón [6] already considered this problem in his foundational paper on the complex method.

Results for the real method have appeared in the last few years. See, for example, the papers by Fernandez and Silva [14], Fernández-Cabrera and Martínez $[12,13]$, and Cobos, Fernández-Cabrera and Martínez [7, 8]. Quantitative results for the real method in terms of the measure of non-compactness have been established by Mastyło and Silva [25] and Besoy and Cobos [5].

In this paper we establish a compactness result for bilinear operators of the kind of the theorem of Janson. Furthermore, as an application of the result, we improve a compactness result of Fernández-Cabrera and Martínez [13] on convolution operators among Lorentz spaces.

In contrast to the majority of the papers on compact bilinear operators and real interpolation, which are based on the ideas developed by Cobos and Peetre [9] and Cobos, Kühn and Schonbek [10] to deal with compact linear operators, our approach here is different. We work assuming an approximation condition on the couple in the target and combining the ideas of Janson [19] with some others of our previous papers.

\section{Preliminaries}

Let $\left(A,\|\cdot\|_{A}\right)$ be a quasi-Banach space and let $c=c_{A} \geq 1$ be the constant in the quasi-triangle inequality. Let $0<p \leq 1$ such that $c=2^{1 / p-1}$. Then it is known that there is another quasi-norm $\||\cdot|\|$ on $A$ which is equivalent to $\|\cdot\|_{A}$ and satisfies that

$$
\left\|\left|a_{1}+a_{2}\right|\right\|^{p} \leq\left\|\left.\left|a_{1}\right|\right|^{p}+\right\|\left|a_{2}\right| \|^{p}, a_{1}, a_{2} \in A
$$


(see $[23, \S 5.10]$ or $[22$, Proposition 1.c.5]). The function $\||\cdot|\|$ is said to be a $p$-norm and $(A,\||\cdot|\|)$ is called a $p$-Banach space. Note that if $A$ is $p$-Banach then it is also $r$-Banach for any $0<r<p$.

Let $\bar{A}=\left(A_{0}, A_{1}\right)$ be a ( $p$-Banach) quasi-Banach couple, that is to say, two ( $p$-Banach) quasi-Banach spaces $A_{0}, A_{1}$ which are continuously embedded in the same Hausdorff topological vector space.

We put $A_{j}^{\circ}$ for the closure of $A_{0} \cap A_{1}$ in the norm of $A_{j}$. It is clear that $\left(A_{0}^{\circ}, A_{1}^{\circ}\right)$ is also a quasi-Banach couple.

Given $t>0$, Peetre's $K$ - and $J$-functional are defined by

$$
K(t, a)=K\left(t, a ; A_{0}, A_{1}\right)=\inf \left\{\left\|a_{0}\right\|_{A_{0}}+t\left\|a_{1}\right\|_{A_{1}}: a=a_{0}+a_{1}, a_{j} \in A_{j}\right\}
$$

where $a \in A_{0}+A_{1}$ and

$$
J(t, a)=J\left(t, a ; A_{0}, A_{1}\right)=\max \left\{\|a\|_{A_{0}}, t\|a\|_{A_{1}}\right\}, \quad a \in A_{0} \cap A_{1} .
$$

The function $K(1, \cdot)$ coincides with the quasi-norm of $A_{0}+A_{1}$ and $J(1, \cdot)$ with the quasi-norm of $A_{0} \cap A_{1}$.

If $\|\cdot\|_{A_{j}}$ is a $p$-norm for $j=0,1$, then $J(t, \cdot)$ is a $p$-norm on $A_{0} \cap A_{1}$ and the functional

$$
K_{p}(t, a)=\inf \left\{\left(\left\|a_{0}\right\|_{A_{0}}^{p}+t^{p}\left\|a_{1}\right\|_{A_{1}}^{p}\right)^{1 / p}: a=a_{0}+a_{1}, a_{j} \in A_{j}\right\}
$$

is a $p$-norm on $A_{0}+A_{1}$ which is equivalent to $K(t, \cdot)$

$$
K(t, a) \leq K_{p}(t, a) \leq 2^{1 / p-1} K(t, a), \quad a \in A_{0}+A_{1}, t>0 .
$$

Let $0<\theta<1$ and $0<q \leq \infty$. The real interpolation space $\bar{A}_{\theta, q}=$ $\left(A_{0}, A_{1}\right)_{\theta, q}$ realized by means of the $K$-functional is formed by all $a \in A_{0}+A_{1}$ having a finite quasi-norm

$$
\|a\|_{\bar{A}_{\theta, q}}= \begin{cases}\left(\sum_{m=-\infty}^{\infty}\left(2^{-\theta m} K\left(2^{m}, a\right)\right)^{q}\right)^{1 / q} & \text { if } \quad 0<q<\infty \\ \sup _{m \in \mathbb{Z}}\left\{2^{-\theta m} K\left(2^{m}, a\right)\right\} & \text { if } \quad q=\infty .\end{cases}
$$

If we replace the number 2 by any other positive number $\rho \neq 1$, then the space $\bar{A}_{\theta, q}$ does not change and the new quasi-norm is equivalent to $\|\cdot\|_{\bar{A}_{\theta, q}}$.

For our later considerations we also define $\bar{A}_{\theta, q}$ for $\theta=0$ or 1 by putting $\bar{A}_{0, q}=A_{0}$ and $\bar{A}_{1, q}=A_{1}$ for any $0<q \leq \infty$.

Subsequently, $\ell_{q}$ stands for the usual space of $q$-summable sequences with $\mathbb{Z}$ as index set.

The space $\bar{A}_{\theta, q}$ realized by means of the $J$-functional is formed by all sums $a=\sum_{m=-\infty}^{\infty} u_{m}$ (convergence in $A_{0}+A_{1}$ ) where $\left(u_{m}\right) \subseteq A_{0} \cap A_{1}$ and $\left(2^{-\theta m} J\left(2^{m}, u_{m}\right)\right) \in \ell_{q}$. The quasi-norm

$$
\|a\|_{\bar{A}_{\theta, q}}^{J}=\inf \left\{\left\|\left(2^{-\theta m} J\left(2^{m}, u_{m}\right)\right)\right\|_{\ell_{q}}: a=\sum_{m=-\infty}^{\infty} u_{m}\right\}
$$

is equivalent to $\|\cdot\|_{\bar{A}_{\theta, q}}$. Again, replacing 2 by any other positive number $\rho \neq 1$, we get the same space with an equivalent quasi-norm. This fact will be of some use later. 
We refer to the books by Bergh and Löfström [4], Triebel [31] and Bennet and Sharpley [1] for other properties of the real interpolation spaces.

Let $A, B, E$ be quasi-Banach spaces and let $T: A \times B \longrightarrow E$ be a bilinear operator. We say that $T$ is bounded if

$$
\|T\|_{A \times B, E}=\sup \left\{\|T(a, b)\|_{E}:\|a\|_{A} \leq 1,\|b\|_{B} \leq 1\right\}<\infty .
$$

We write $\mathcal{L}(A \times B, E)$ for the space of all bounded bilinear operators from $A \times B$ into $E$. We say that the operator $T$ is compact if for any bounded sets $V \subseteq A$ and $W \subseteq B$, the closure of $T(V, W)=\{T(a, b): a \in V, b \in W\}$ is compact in $E$. This is equivalent to ask that $T\left(U_{A}, U_{B}\right)$ is precompact in $E$. Here $U_{A}$ stands for the closed unit ball of $A$ and $U_{B}$ for the closed unit ball of $B$. Compactness is also equivalent to the fact that for any bounded sequences $\left(a_{n}\right) \subseteq A,\left(b_{n}\right) \subseteq B$, the sequence $\left(T\left(a_{n}, b_{n}\right)\right)$ has a convergent subsequence. We write $\mathcal{K}(A \times B, E)$ for the collection of all compact bilinear operators from $A \times B$ into $E$.

Let $T \in \mathcal{K}(A \times B, E)$, let $Y$ be a quasi-Banach space and $R \in \mathcal{L}(E, Y)$, then clearly $R T=R \circ T$ belongs to $\mathcal{K}(A \times B, Y)$. On the other hand, if $T \in \mathcal{L}(A \times B, E)$ and $S \in \mathcal{L}(E, Y)$ is compact, then $S T \in \mathcal{K}(A \times B, Y)$.

Another useful property of compact bilinear operators is that if $\left(T_{n}\right) \subseteq$ $\mathcal{K}(A \times B, E)$ is a convergent sequence in $\mathcal{L}(A \times B, E)$ to the operator $T$, then $T \in \mathcal{K}(A \times B, E)$.

Let $\bar{A}=\left(A_{0}, A_{1}\right), \bar{B}=\left(B_{0}, B_{1}\right), \bar{E}=\left(E_{0}, E_{1}\right)$ be quasi-Banach couples. By $\mathcal{B}(\bar{A} \times \bar{B}, \bar{E})$ we designate the collection of all bilinear operators $T$ defined from $\left(A_{0} \cap A_{1}\right) \times\left(B_{0} \cap B_{1}\right)$ into $E_{0} \cap E_{1}$ such that there are constants $M_{j}>0$ such that

$$
\|T(a, b)\|_{E_{j}} \leq M_{j}\|a\|_{A_{j}}\|b\|_{B_{j}}, a \in A_{0} \cap A_{1}, b \in B_{0} \cap B_{1}, j=0,1 .
$$

If $T \in \mathcal{B}(\bar{A} \times \bar{B}, \bar{E})$ it is not hard to check that $T$ may be uniquely extended to a bounded bilinear operator $T_{j}: A_{j}^{\circ} \times B_{j}^{\circ} \longrightarrow E_{j}$ with

$$
\|T\|_{j}:=\left\|T_{j}\right\|_{A_{j}^{\circ} \times B_{j}^{\circ}, E_{j}}=M_{j} \quad j=0,1 .
$$

We say that $T: A_{j}^{\circ} \times B_{j}^{\circ} \longrightarrow E_{j}$ is compact if $T_{j}$ does it.

Definition 2.1. Let $\bar{A}, \bar{B}, \bar{E}$ be quasi-Banach couples, let $T$ be a bilinear operator and let $\alpha_{0}, \alpha_{1}, \alpha_{2} \in \mathbb{R}$ with $\alpha_{1} \neq 0$ and $\alpha_{2} \neq 0$. The set $\Omega=$ $\Omega\left(\bar{A}, \bar{B}, \bar{E}, T, \alpha_{0}, \alpha_{1}, \alpha_{2}\right)$ consists of all couples $\bar{\theta}=\left(\theta_{1}, \theta_{2}\right) \in[0,1]^{2}$ satisfying that

(2.1) the number $\theta:=\alpha_{0}+\alpha_{1} \theta_{1}+\alpha_{2} \theta_{2}$ belongs to $[0,1]$

and

(2.2) there is a triple $\bar{r}=\left(r_{1}, r_{2}, r\right) \in(0, \infty]^{3}$ and a positive constant $M>0$ such that $\|T(a, b)\|_{\bar{E}_{\theta, r}} \leq M\|a\|_{\bar{A}_{\theta_{1}, r_{1}}}\|b\|_{\bar{B}_{\theta_{2}, r_{2}}}$ for any $a \in A_{0} \cap A_{1}, b \in$ $B_{0} \cap B_{1}$.

Note that in Definition 2.1 the parameters $\theta_{1}, \theta_{2}, \theta$ may take the limit values 0 and 1 . As we have defined before, in these limit cases we put $\bar{A}_{\theta, q}=A_{\theta}$ and similarly for $\bar{B}$ and $\bar{E}$.

Janson has shown in [19, Theorem 1] that the set $\Omega$ is convex.

If $\bar{\theta} \in \Omega$ and $\bar{r}$ satisfies (2.2) with $\bar{\theta}$, there may be other triples that also satisfy (2.2) with $\bar{\theta}$. For example, if $\bar{E}_{\theta, \infty}$ is $p$-Banach then it follows from [19, Lemma 2] that $(p, p, \infty)$ satisfies $(2.2)$ with $\bar{\theta}$. 
Janson has proved in [19, Theorem 2] that if $\bar{\theta}$ belongs to the interior of $\Omega$, $0<q, q_{1}, q_{2} \leq \infty$ and $1 / q=1 / q_{1}+1 / q_{2}$, then there is a positive constant $M>0$ such that

$$
\|T(a, b)\|_{\bar{E}_{\theta, q}} \leq M\|a\|_{\bar{A}_{\theta_{1}, q_{1}}}\|b\|_{\bar{B}_{\theta_{2}, q_{2}}} \text { for any } a \in A_{0} \cap A_{1}, b \in B_{0} \cap B_{1} .
$$

In the next section we establish a compactness result of this type.

\section{The compactness theorem}

In what follows we assume that $\bar{E}$ satisfies the following approximation condition (see the papers by Fernández-Cabrera and Martínez [13, p. 1196], Calderón $[6, \S 10.4]$, Persson [27, p. 216] and Edmunds and Teixeira [29, p. 133]).

Definition 3.1. We say that a quasi-Banach couple $\bar{E}=\left(E_{0}, E_{1}\right)$ satisfies condition $(\mathfrak{H})$ if there is a family of operators $\left\{P_{\lambda}\right\}_{\lambda \in \Lambda} \subseteq \mathcal{L}\left(E_{0}+E_{1}, E_{0} \cap E_{1}\right)$ and a constant $C>0$ such that

$$
\begin{gathered}
P_{\lambda}: E_{0}+E_{1} \longrightarrow E_{0} \cap E_{1} \quad \text { is compact, } \lambda \in \Lambda . \\
\left\|P_{\lambda}\right\|_{E_{j}, E_{j}} \leq C, \quad j=0,1, \quad \lambda \in \Lambda .
\end{gathered}
$$

(3.3) For every compact subset $K \subseteq E_{0}$ and every $\varepsilon>0$,

there is $\lambda_{0} \in \Lambda$ such that $\left\|x-P_{\lambda_{0}} x\right\|_{E_{0}} \leq \varepsilon$ for every $x \in K$.

Next we give examples of quasi-Banach couples satisfying $(\mathfrak{H})$. Let $(\Omega, \mu)$ be a $\sigma$-finite measure space. For $0<p<\infty$ and $0<q \leq \infty$, recall that the Lorentz space $L_{p, q}(\Omega)$ is formed by all (equivalent classes of) measurable functions which have a finite quasi-norm

$$
\|f\|_{L_{p, q}(\Omega)}=\left(\int_{0}^{\infty}\left(t^{1 / p} f^{*}(t)\right)^{q} \frac{d t}{t}\right)^{1 / q}
$$

(the integral should be replaced by the supremum if $q=\infty$ ). Here $f^{*}$ is the non-increasing rearrangement of $f$

$$
f^{*}(t)=\inf \{s>0: \mu\{x \in \Omega:|f(x)|>s\} \leq t\} .
$$

See $[18,1,11]$. If $p=q$ then we recover the Lebesgue space $L_{p}(\Omega)$.

For $1<p<\infty, 0<q \leq \infty$ and $\theta=1-1 / p$, it follows from [4, Theorem 5.2.1] that we have with equivalent quasi-norms

$$
\left(L_{1}(\Omega), L_{\infty}(\Omega)\right)_{\theta, q}=L_{p, q}(\Omega) .
$$

We also recall that for $0<p<\infty$ and $0<q \leq r \leq \infty$, the space $L_{p, q}(\Omega)$ is continuously embedded in $L_{p, r}(\Omega)$.

Example 3.2. Let $(\Omega, \mu)$ be a $\sigma$-finite measure space and let $1<p_{0}, p_{1}<\infty$, $0<q_{0}<\infty$ and $0<q_{1} \leq \infty$. Then $\left(L_{p_{0}, q_{0}}(\Omega), L_{p_{1}, q_{1}}(\Omega)\right)$ satisfy $(\mathfrak{H})$. Indeed, according to $(3.4)$, spaces $L_{p_{j}, q_{j}}(\Omega)$ are interpolation spaces with respect to the couple $\left(L_{1}(\Omega), L_{\infty}(\Omega)\right)$. Moreover, simple functions are dense in $L_{p_{0}, q_{0}}(\Omega)$ 
because $q_{0}<\infty$ (see $[18,(2.4)$, p. 258]). Whence, the arguments given in [13, Proposition 4.5] are still valid in this case. The family of operators is

$$
P f=\sum_{k=1}^{L}\left(\mu\left(O_{k}\right)^{-1} \int_{\Omega} f \chi_{O_{k}} d \mu\right) \chi_{O_{k}}
$$

where $O_{1}, \ldots, O_{L}$ is any finite collection of pairwise disjoint measurable sets of finite measure.

Example 3.3. Let $E_{0}$ be a quasi-Banach space and let $\left(x_{m}\right)$ be a basis of $E_{0}$, that is, for any $x \in E_{0}$ there is a unique sequence of scalars $\left(\lambda_{m}\right)$ such that $x=\sum_{m=1}^{\infty} \lambda_{m} x_{m}$. Put $f_{m}(x)=\lambda_{m}$. It turns out that the functionals $f_{m}$ are bounded on $E_{0}$ (see [33, Proposition 6.35]). Hence, $\left(x_{m}, f_{m}\right)$ is a Schauder basis on $E_{0}$. Consider the projections associated to the basis

$$
P_{m} x=\sum_{j=1}^{m} f_{j}(x) x_{j}, \quad m \in \mathbb{N} .
$$

It follows from Banach-Steinhaus theorem [23, p. 169] that

$$
\sup \left\{\left\|P_{m}\right\|_{E_{0}, E_{0}}: m \in \mathbb{N}\right\}<\infty \text {. }
$$

Now let $E_{1}$ be another quasi-Banach space such that $\left(E_{0}, E_{1}\right)$ is a quasiBanach couple and $\left(x_{m}\right)$ is also a basis on $E_{1}$. Then, for the couple $\left(E_{0}, E_{1}\right)$, the sequence $\left(P_{m}\right)$ satisfies properties (3.1) to (3.3) in Definition 3.1. Indeed, it is not hard to check that $P_{m} \in \mathcal{L}\left(E_{0}+E_{1}, E_{0} \cap E_{1}\right)$ and they are compact because the dimension of the range of $P_{m}$ is finite. Furthermore, given any compact subset $K \subseteq E_{0}$ and any $\varepsilon>0$, there is a finite set $\left\{z_{1}, \cdots, z_{r}\right\} \subseteq E_{0}$ such that

$$
K \subseteq \bigcup_{j=1}^{r}\left\{z_{j}+\frac{\varepsilon}{3 L} U_{E_{0}}\right\}
$$

with $L=c_{E_{0}}+c_{E_{0}}^{2} C$, where $c_{E_{0}}$ is the constant in the quasi-triangle inequality in $E_{0}$ and $C$ is the constant in (3.2). Since the set $\left\{z_{j}\right\}_{j=1}^{r}$ is finite, we can take $N \in \mathbb{N}$ such that

$$
\left\|z_{j}-P_{N} z_{j}\right\|_{E_{0}} \leq \frac{\varepsilon}{3 c_{E_{0}}^{2}}, \quad j=1,2, \cdots, r
$$

Given any $x \in K$, we can find $j$ with $\left\|z-z_{j}\right\| \leq \varepsilon / 3 L$ and so

$$
\begin{aligned}
\left\|x-P_{N} x\right\|_{E_{0}} & \leq c_{E_{0}}\left\|x-z_{j}\right\|_{E_{0}}+c_{E_{0}}^{2}\left\|z_{j}-P_{N} z_{j}\right\|_{E_{0}}+c_{E_{0}}^{2}\left\|P_{N} z_{j}-P_{N} x\right\|_{E_{0}} \\
& \leq c_{E_{0}} \frac{\varepsilon}{3 L}+\frac{\varepsilon}{3}+\frac{c_{E_{0}}^{2} C \varepsilon}{3 L} \leq \varepsilon .
\end{aligned}
$$

In particular, if $0<p_{0}, p_{1}<\infty$ then the couple $\left(\ell_{p_{0}}, \ell_{p_{1}}\right)$ satisfies $(\mathfrak{H})$. Other concrete examples are couples of Triebel-Lizorkin spaces $F_{p, q}^{s}\left(\mathbb{R}^{n}\right)$ and couples of Besov spaces $B_{p, q}^{s}\left(\mathbb{R}^{n}\right)$ (see $[31,32]$ ). Since these spaces have a common basis formed by wavelets (see [32, Theorem 3.5]), we obtain that for $0<$ $p_{0}, p_{1}, q_{0}, q_{1}<\infty$ and $-\infty<s_{0}, s_{1}<\infty$, the couples $\left(F_{p_{0}, q_{0}}^{s_{0}}\left(\mathbb{R}^{n}\right), F_{p_{1}, q_{1}}^{s_{1}}\left(\mathbb{R}^{n}\right)\right)$ and $\left(B_{p_{0}, q_{0}}^{s_{0}}(\Omega), B_{p_{1}, q_{1}}^{s_{1}}(\Omega)\right)$ satisfy $(\mathfrak{H})$. 
Now suppose that $H_{0}$ and $H_{1}$ are separable Hilbert spaces and let $S_{p, q}\left(H_{0}, H_{1}\right)$ be the space of all bounded linear operators $T \in \mathcal{L}\left(H_{0}, H_{1}\right)$ whose singular numbers belong to the Lorentz sequence space $\ell_{p, q}($ see $[30,16,22,28])$. If $1<p<\infty$ and $0<q<\infty$, then spaces $S_{p, q}\left(H_{0}, H_{1}\right)$ have a common basis. This was proved in [15, Proposition 3.1] for the Banach case $1 \leq q<\infty$. The same arguments work also for $0<q<1$. Consequently, if $1<p_{0}, p_{1}<\infty$ and $0<q_{0}, q_{1}<\infty$, the couple $\left(S_{p_{0}, q_{0}}\left(H_{0}, H_{1}\right), S_{p_{1}, q_{1}}\left(H_{0}, H_{1}\right)\right)$ satisfies $(\mathfrak{H})$.

Next we show that condition $(\mathfrak{H})$ allows to approximate certain bilinear operators $T$ by operators $P_{\lambda} T$.

Proposition 3.4. Let $\bar{A}=\left(A_{0}, A_{1}\right), \bar{B}=\left(B_{0}, B_{1}\right), \bar{E}=\left(E_{0}, E_{1}\right)$ be quasiBanach couples and let $T \in \mathcal{B}(\bar{A} \times \bar{B}, \bar{E})$ with $T: A_{0}^{\circ} \times B_{0}^{\circ} \longrightarrow E_{0}$ compactly. Assume that $\bar{E}$ satisfies condition ( $\mathfrak{H})$. Let $0<\theta<1,1 \leq p, q<\infty$ and $1 \leq r \leq \infty$ with $1 / p+1 / q=1+1 / r$. Then for any $\varepsilon>0$, there $i s \in \Lambda$ such that

$$
\left\|\left(T-P_{\lambda} T\right)(a, b)\right\|_{\bar{E}_{\theta, r}} \leq \varepsilon\|a\|_{\bar{A}_{\theta, p}}\|b\|_{\bar{B}_{\theta, q}} \text { for any } a \in A_{0} \cap A_{1}, b \in B_{0} \cap B_{1} .
$$

Proof. Proceeding as in the proof of [13, Theorem 4.1], we get that there is a constant $c_{1}$ independent of $T$ such that for any $\lambda \in \Lambda, a \in A_{0} \cap A_{1}$ and $b \in B_{0} \cap B_{1}$ we have that

$$
\left\|\left(T-P_{\lambda} T\right)(a, b)\right\|_{\bar{E}_{\theta, r}} \leq c_{1}\left\|T-P_{\lambda} T\right\|_{0}^{1-\theta}\left\|T-P_{\lambda} T\right\|_{1}^{\theta}\|a\|_{\bar{A}_{\theta, p}}\|b\|_{\bar{B}_{\theta, q}} .
$$

By (3.2), we obtain

$$
\begin{aligned}
\left\|T-P_{\lambda} T\right\|_{1} & \leq\left\|I-P_{\lambda}\right\|_{E_{1}, E_{1}}\|T\|_{1} \\
& \leq c_{E_{1}}\left(1+\left\|P_{\lambda}\right\|_{E_{1}, E_{1}}\right)\|T\|_{1} \\
& \leq c_{E_{1}}(1+C)\|T\|_{1}=c_{2} .
\end{aligned}
$$

Moreover, using compactness of $T: A_{0}^{\circ} \times B_{0}^{\circ} \longrightarrow E_{0}$ and (3.3), we can choose $\lambda \in \Lambda$ such that

$$
\left\|T-P_{\lambda} T\right\|_{0} \leq\left(\frac{\varepsilon}{c_{1} c_{2}^{\theta}}\right)^{1 / 1-\theta} .
$$

Combining these three facts the result follows.

Our next step is to connect $(\mathfrak{H})$ with a given operator $T$ and the set $\Omega$.

Definition 3.5. Let $\bar{A}=\left(A_{0}, A_{1}\right), \bar{B}=\left(B_{0}, B_{1}\right), \bar{E}=\left(E_{0}, E_{1}\right)$ be quasiBanach couples, let $T$ be a bilinear operator and let $\alpha_{0}, \alpha_{1}, \alpha_{2} \in \mathbb{R}$, with $\alpha_{1} \neq 0$, $\alpha_{2} \neq 0$. Consider the set $\Omega=\Omega\left(\bar{A}, \bar{B}, \bar{E}, T, \alpha_{0}, \alpha_{1}, \alpha_{2}\right)$ given in Definition 2.1.

We say that $\bar{E}, T$ and $\Omega$ satisfy the condition $(\mathcal{H})$ if $\bar{E}$ satisfy $(\mathfrak{H})$ with a family of operators $\left\{P_{\lambda}\right\}_{\lambda \in \Lambda}$ such that in addition to (3.1), (3.2) and (3.3), operators $\left\{P_{\lambda}\right\}_{\lambda \in \Lambda}$ satisfy

(3.7) For any $\varepsilon>0$ and for any finite subset $\left\{\bar{\theta}_{k}=\left(\theta_{1, k}, \theta_{2, k}\right)\right\}_{k=1}^{m}$ in the interior of $\Omega$, there exist a set of triples $\left\{\bar{r}_{k}=\left(r_{1, k}, r_{2, k}, r_{k}\right\}_{k=1}^{m}\right.$ where $\bar{r}_{k}$ and $\bar{\theta}_{k}$ satisfy (2.2) with $\theta_{k}=\alpha_{0}+\alpha_{1} \theta_{1, k}+\alpha_{2} \theta_{2, k}$ and there is $\lambda \in \Lambda$ such that

$$
\left\|\left(T-P_{\lambda} T\right)(a, b)\right\|_{\bar{E}_{\theta_{k}, r_{k}}} \leq \varepsilon\|a\|_{\bar{A}_{\theta_{1, k}, r_{1, k}}}\|b\|_{\bar{B}_{\theta_{2, k}, r_{2, k}}}
$$

for any $a \in A_{0} \cap A_{1}, \quad b \in B_{0} \cap B_{1}$ and $1 \leq k \leq m$. 
Next we establish the main result of the paper.

Theorem 3.6. Let $\bar{A}=\left(A_{0}, A_{1}\right), \bar{B}=\left(B_{0}, B_{1}\right), \bar{E}=\left(E_{0}, E_{1}\right)$ be quasi-Banach couples, let $T$ be a bilinear operator and let $\alpha_{0}, \alpha_{1}, \alpha_{2} \in \mathbb{R}$, with $\alpha_{1} \neq 0$ and $\alpha_{2} \neq 0$. Consider the set $\Omega=\Omega\left(\bar{A}, \bar{B}, \bar{E}, T, \alpha_{0}, \alpha_{1}, \alpha_{2}\right)$ given in Definition 2.1 and assume that $\bar{E}, T$ and $\Omega$ satisfy the condition $(\mathcal{H})$ with the family of operators $\left\{P_{\lambda}\right\}_{\lambda \in \Lambda}$.

If $\bar{\theta}=\left(\theta_{1}, \theta_{2}\right)$ belongs to the interior of $\Omega$ and $\theta=\alpha_{0}+\alpha_{1} \theta_{1}+\alpha_{2} \theta_{2}$, then for any $0<q_{1}, q_{2}, q \leq \infty$ with $1 / q=1 / q_{1}+1 / q_{2}$, there is a constant $L>0$ such that for any $\varepsilon>0$ there is $\lambda \in \Lambda$ such that

$\left\|\left(T-P_{\lambda} T\right)(a, b)\right\|_{\bar{E}_{\theta, q}} \leq L \varepsilon\|a\|_{\bar{A}_{\theta_{1}, q_{1}}}\|b\|_{\bar{B}_{\theta_{2}, q_{2}}}$ for any $a \in A_{0} \cap A_{1}, b \in B_{0} \cap B_{1}$.

Proof. Our arguments use some ideas introduced by Janson [19]. Since $\bar{\theta}=$ $\left(\theta_{1}, \theta_{2}\right)$ belongs to the interior of $\Omega$, taking $\delta>0$ sufficiently small, the four couples

$$
\begin{array}{ll}
\left(\theta_{1}+\delta / \alpha_{1}, \theta_{2}+\delta / \alpha_{2}\right), & \left(\theta_{1}-\delta / \alpha_{1}, \theta_{2}+\delta / \alpha_{2}\right) \\
\left(\theta_{1}+\delta / \alpha_{1}, \theta_{2}-\delta / \alpha_{2}\right), & \left(\theta_{1}-\delta / \alpha_{1}, \theta_{2}-\delta / \alpha_{2}\right)
\end{array}
$$

belong to the interior of $\Omega$. Let $\bar{\eta}=\left(\eta_{1}, \eta_{2}\right)$ be any of these four couples and $\eta=\alpha_{0}+\alpha_{1} \eta_{1}+\alpha_{2} \eta_{2}$. Given any $\varepsilon>0$, by (3.7), there is a triple $\bar{s}=\bar{s}(\bar{\eta})=$ $\left(s_{1}, s_{2}, s\right)$ satisfying $(2.2)$ for $\bar{\eta}$ and there is $\lambda \in \Lambda$ such that for $S_{\lambda}:=T-P_{\lambda} T$ we have

$$
\left\|S_{\lambda}(a, b)\right\|_{\bar{E}_{\eta, s}} \leq \varepsilon\|a\|_{\bar{A}_{\eta_{1}, s_{1}}}\|b\|_{\bar{B}_{\eta_{2}, s_{2}}} \text {, for any } a \in A_{0} \cap A_{1}, b \in B_{0} \cap B_{1} .
$$

The same $\lambda$ satisfying (3.9) for any of the four couples $\bar{\eta}$.

The space $\bar{E}_{\eta, s}$ is continuously embedded in $\bar{E}_{\eta, \infty}$. Besides, there are positive constants $d_{1}, d_{2}>0$ such that

$$
\|a\|_{\bar{A}_{\eta_{1}, s_{1}}} \leq d_{1}\|a\|_{A_{0}}^{1-\eta_{1}}\|a\|_{A_{1}}^{\eta_{1}}, \quad a \in A_{0} \cap A_{1}
$$

and

$$
\|b\|_{\bar{B}_{\eta_{2}, s_{2}}} \leq d_{2}\|b\|_{B_{0}}^{1-\eta_{2}}\|b\|_{B_{1}}^{\eta_{2}}, \quad b \in B_{0} \cap B_{1}
$$

(see [4, Theorem 3.11.2]). Combining these facts with (3.9) we obtain that there is a positive constant $c_{1}>0$ such that for any $t>0$ we have

$$
K\left(t, S_{\lambda}(a, b)\right) \leq c_{1} \varepsilon t^{\eta}\|a\|_{A_{0}}^{1-\eta_{1}}\|a\|_{A_{1}}^{\eta_{1}}\|b\|_{B_{0}}^{1-\eta_{2}}\|b\|_{B_{1}}^{\eta_{2}} .
$$

In what follows we think that the spaces $\bar{A}_{\theta_{1}, q_{1}}$ and $\bar{B}_{\theta_{2}, q_{2}}$ are realized by the $J$-method where the basis is not 2 but $2^{\alpha_{1}}$ and $2^{\alpha_{2}}$, respectively. Take any $a \in A_{0} \cap A_{1}, \quad b \in B_{0} \cap B_{1}$. According to [19, Lemma 1] we can find $J$ representations $a=\sum_{j=-N}^{N} a_{j}, b=\sum_{k=-N}^{N} b_{k}$ with only a finite number of elements such that for

$$
x_{j}=2^{-\theta_{1} \alpha_{1} j} J\left(2^{\alpha_{1} j}, a_{j}\right) \text { and } y_{k}=2^{-\theta_{2} \alpha_{2} k} J\left(2^{\alpha_{2} k}, b_{k}\right)
$$

we have

$$
\left\|\left(x_{j}\right)\right\|_{\ell_{q_{1}}} \leq c_{2}\|a\|_{\bar{A}_{\theta_{1}, q_{1}}} \text { and }\left\|\left(y_{k}\right)\right\|_{\ell_{q_{2}}} \leq c_{2}\|b\|_{\bar{B}_{\theta_{2}, q_{2}}}
$$


where the constant $c_{2}>0$ is independent of $a$ and $b$. Take any $(j, k) \in \mathbb{Z}^{2}$. By (3.10) we have that

$$
\begin{aligned}
t^{-\theta} K\left(t, S_{\lambda}\left(a_{j}, b_{k}\right)\right) & \leq c_{1} \varepsilon t^{\eta-\theta}\left\|a_{j}\right\|_{A_{0}}^{1-\eta_{1}}\left\|a_{j}\right\|_{A_{1}}^{\eta_{1}}\left\|b_{k}\right\|_{B_{0}}^{1-\eta_{2}}\left\|b_{k}\right\|_{B_{1}}^{\eta_{2}} \\
& \leq c_{1} \varepsilon t^{\eta-\theta} 2^{-\eta_{1} \alpha_{1} j} J\left(2^{\alpha_{1} j}, a_{j}\right) 2^{-\eta_{2} \alpha_{2} k} J\left(2^{\alpha_{2} k}, b_{k}\right) \\
& \leq c_{1} \varepsilon t^{\alpha_{1}\left(\eta_{1}-\theta_{1}\right)} 2^{\left(\theta_{1}-\eta_{1}\right) \alpha_{1} j} x_{j} t^{\alpha_{2}\left(\eta_{2}-\theta_{2}\right)} 2^{\left(\theta_{2}-\eta_{2}\right) \alpha_{2} k} y_{k} .
\end{aligned}
$$

Let $m \in \mathbb{Z}$ and put $t=2^{m}$. We derive that

$$
2^{-m \theta} K\left(2^{m}, S_{\lambda}\left(a_{j}, b_{k}\right)\right) \leq c_{1} \varepsilon 2^{\left(\theta_{1}-\eta_{1}\right) \alpha_{1}(j-m)} x_{j} 2^{\left(\theta_{2}-\eta_{2}\right) \alpha_{2}(k-m)} y_{k} .
$$

Since this holds for any of the four couples $\bar{\eta}$ in (3.8), given any $m \in \mathbb{Z}$ we can choose $\bar{\eta}$ such that

$$
2^{-m \theta} K\left(2^{m}, S_{\lambda}\left(a_{j}, b_{k}\right)\right) \leq c_{1} \varepsilon 2^{-\delta|j-m|-\delta|k-m|} x_{j} y_{k} .
$$

Now applying Hölder's inequality we get

$$
\begin{aligned}
\|\left(2 ^ { - m \theta } K \left(2^{m}\right.\right. & \left.\left., S_{\lambda}\left(a_{j+m}, b_{k+m}\right)\right)\right)_{m}\left\|_{\ell_{q}} \leq c_{1} \varepsilon 2^{-\delta|j|-\delta|k|}\right\|\left(x_{j+m} y_{k+m}\right)_{m} \|_{\ell_{q}} \\
& \leq c_{1} \varepsilon 2^{-\delta|j|-\delta|k|}\left\|\left(x_{j+m}\right)_{m}\right\|_{\ell_{q_{1}}}\left\|\left(y_{j+m}\right)_{m}\right\|_{\ell_{q_{2}}} \\
& \leq c_{1} c_{2}^{2} \varepsilon 2^{-\delta|j|-\delta|k|}\|a\|_{\bar{A}_{\theta_{1}, q_{1}}}\|b\|_{\bar{B}_{\theta_{2}, q_{2}}} .
\end{aligned}
$$

where we have used (3.11) in the last inequality. Choose $0<p \leq 1$ such that $E_{0}$ and $E_{1}$ are $p$-Banach spaces and $p \leq q$. According to Minkowski's inequality and (3.12), we derive

$$
\begin{aligned}
\left\|S_{\lambda}(a, b)\right\|_{\bar{E}_{\theta, q}} & =\left\|\left(2^{-\theta m p} K\left(2^{m}, S_{\lambda}(a, b)\right)^{p}\right)^{1 / p}\right\|_{\ell_{q}} \\
& \leq c_{3}\left\|\left(2^{-\theta m p} \sum_{(j, k) \in \mathbb{Z}^{2}} K\left(2^{m}, S_{\lambda}\left(a_{j+m}, b_{k+m}\right)\right)^{p}\right)\right\|_{\ell_{q / p}}^{1 / p} \\
& \leq c_{3}\left(\sum_{(j, k) \in \mathbb{Z}^{2}}\left\|\left(2^{-\theta m} K\left(2^{m}, S_{\lambda}\left(a_{j+m}, b_{k+m}\right)\right)\right)\right\|_{\ell_{q}}^{p}\right)^{1 / p} \\
& \leq c_{4} \varepsilon\left(\sum_{(j, k) \in \mathbb{Z}^{2}} 2^{(-\delta|j|-\delta|k|) p}\right)^{1 / p}\|a\|_{\bar{A}_{\theta_{1}, q_{1}}}\|b\|_{\bar{B}_{\theta_{2}, q_{2}}} \\
& =L \varepsilon\|a\|_{\bar{A}_{\theta_{1}, q_{1}}}\|b\|_{\bar{B}_{\theta_{2}, q_{2}}} .
\end{aligned}
$$

This completes the proof.

Theorem 3.7. Let $\bar{A}=\left(A_{0}, A_{1}\right), \bar{B}=\left(B_{0}, B_{1}\right), \bar{E}=\left(E_{0}, E_{1}\right)$ be quasi-Banach couples, let $T$ be a bilinear operator and let $\alpha_{0}, \alpha_{1}, \alpha_{2} \in \mathbb{R}$, with $\alpha_{1} \neq 0$ and $\alpha_{2} \neq 0$. Consider the set $\Omega=\Omega\left(\bar{A}, \bar{B}, \bar{E}, T, \alpha_{0}, \alpha_{1}, \alpha_{2}\right)$ and assume that $\bar{E}, T$, $\Omega$ satisfy $(\mathcal{H})$.

If $\bar{\theta}=\left(\theta_{1}, \theta_{2}\right)$ belongs to the interior of $\Omega$ and $\theta=\alpha_{0}+\alpha_{1} \theta_{1}+\alpha_{2} \theta_{2}$, then for any $0<q_{1}, q_{2}, q<\infty$ with $1 / q=1 / q_{1}+1 / q_{2}$, the operator $T$ may be uniquely extended to a compact bilinear operator from $\bar{A}_{\theta_{1}, q_{1}} \times \bar{B}_{\theta_{2}, q_{2}}$ to $\bar{E}_{\theta, q}$.

Proof. Since $q_{1}<\infty$ and $q_{2}<\infty, A_{0} \cap A_{1}$ is dense in $\bar{A}_{\theta_{1}, q_{1}}$ and $B_{0} \cap B_{1}$ in $\bar{B}_{\theta_{2}, q_{2}}$. It follows from [19, Theorem 2] that $T$ may be uniquely extended to a bounded bilinear operator $T: \bar{A}_{\theta_{1}, q_{1}} \times \bar{B}_{\theta_{2}, q_{2}} \longrightarrow \bar{E}_{\theta, q}$. The same happens with $P_{\lambda} T$ for any $\lambda \in \Lambda$. Furthermore, by (3.1), operators $P_{\lambda} T$ are compact. Theorem 3.6 yields that $T$ is the limit of a sequence of operators $P_{\lambda} T$, therefore $T: \bar{A}_{\theta_{1}, q_{1}} \times \bar{B}_{\theta_{2}, q_{2}} \longrightarrow \bar{E}_{\theta, q}$ compactly. 


\section{Compactness of convolution operators}

Fernández-Cabrera and Martínez [13, Theorem 5.3], established a compactness theorem for convolution operators among Lorentz spaces. With the help of the previous results we are going to improve it. The improvement is in the second indices of the Lorentz spaces, replacing the condition $1 / \alpha+1 / \beta=1+1 / \gamma$ used in [13] by $1 / \alpha+1 / \beta=1 / \gamma$.

Subsequently, $\left(\Gamma_{k}, \mu_{k}\right)$ is a $\sigma$-finite measure space for $k=0,1,2$, and $T$ is a bilinear operator satisfying

$$
\left\{\begin{array}{l}
\|T(f, g)\|_{L_{1}\left(\Gamma_{2}\right)} \leq\|f\|_{L_{1}\left(\Gamma_{0}\right)}\|g\|_{L_{1}\left(\Gamma_{1}\right)}, \\
\|T(f, g)\|_{L_{\infty}\left(\Gamma_{2}\right)} \leq\|f\|_{L_{\infty}\left(\Gamma_{0}\right)}\|g\|_{L_{1}\left(\Gamma_{1}\right)}, \\
\|T(f, g)\|_{L_{\infty}\left(\Gamma_{2}\right)} \leq\|f\|_{L_{1}\left(\Gamma_{0}\right)}\|g\|_{L_{\infty}\left(\Gamma_{1}\right)}
\end{array}\right.
$$

This kind of operators are called convolution operators in $[26,1]$.

Theorem 4.1. Suppose $1<p, q, r<\infty, 0<\alpha, \beta, \gamma<\infty$, with $1 / p+1 / q=$ $1+1 / r$ and $1 / \alpha+1 / \beta=1 / \gamma$. Let $T$ be a bilinear operator satisfying (4.1) and such that $T: L_{1}\left(\Gamma_{0}\right) \times L_{1}\left(\Gamma_{1}\right) \longrightarrow L_{1}\left(\Gamma_{2}\right)$ is compact. Then $T$ may be uniquely extended to a compact bilinear operator from $L_{p, \alpha}\left(\Gamma_{0}\right) \times L_{q, \beta}\left(\Gamma_{1}\right)$ to $L_{r, \gamma}\left(\Gamma_{2}\right)$.

Proof. Choose $\bar{A}=\left(L_{1}\left(\Gamma_{0}\right), L_{\infty}\left(\Gamma_{0}\right)\right), \bar{B}=\left(L_{1}\left(\Gamma_{1}\right), L_{\infty}\left(\Gamma_{1}\right)\right)$, $\bar{E}=\left(L_{1}\left(\Gamma_{2}\right), L_{\infty}\left(\Gamma_{2}\right)\right), \alpha_{0}=0, \alpha_{1}=\alpha_{2}=1$ and consider the set $\Omega=$ $\Omega\left(\bar{A}, \bar{B}, \bar{E}, T, \alpha_{0}, \alpha_{1}, \alpha_{2}\right)$ given in Definition 2.1. By (4.1), the points $(0,0)$, $(1,0)$ and $(0,1)$ belong to $\Omega$. Moreover, given any $\bar{\theta}=\left(\theta_{1}, \theta_{2}\right) \in \Omega$, we have that $0 \leq \theta_{1}, \theta_{2} \leq 1$ and $\theta=\alpha_{0}+\alpha_{1} \theta_{1}+\alpha_{2} \theta_{2}=\theta_{1}+\theta_{2} \leq 1$. This shows that $\Omega$ is equal to the triangle in the plain with vertices $(0,0),(1,0),(0,1)$.

According to $[12$, Proposition 4.1], the couple $\bar{E}$ satisfies condition $(\mathfrak{H})$ with the family of operators (3.5). Next we check that (3.7) also holds. We start with the case when the set has only one element.

Let $\bar{\theta}=\left(\theta_{1}, \theta_{2}\right)$ be in the interior of $\Omega$ and let $\theta=\theta_{1}+\theta_{2}$. Put $1 / u=1-\theta_{1}$, $1 / v=1-\theta_{2}, 1 / w=1-\theta$. So $1<u, v, w<\infty$ and $1 / u+1 / v=1+1 / w$. Take any $1 \leq \eta_{1}, \eta_{2}<\infty$ and $1 \leq \eta \leq \infty$ with $1 / \eta_{1}+1 / \eta_{2}=1+1 / \eta$. By (3.4), we have

$$
\left\{\begin{array}{l}
\bar{A}_{\theta_{1}, \eta_{1}}=\left(L_{1}\left(\Gamma_{0}\right), L_{\infty}\left(\Gamma_{0}\right)\right)_{\theta_{1}, \eta_{1}}=L_{u, \eta_{1}}\left(\Gamma_{0}\right), \\
\bar{B}_{\theta_{2}, \eta_{2}}=\left(L_{1}\left(\Gamma_{1}\right), L_{\infty}\left(\Gamma_{1}\right)\right)_{\theta_{2}, \eta_{2}}=L_{v, \eta_{2}}\left(\Gamma_{1}\right), \\
\bar{E}_{\theta, \eta}=\left(L_{1}\left(\Gamma_{2}\right), L_{\infty}\left(\Gamma_{2}\right)\right)_{\theta, \eta}=L_{w, \eta}\left(\Gamma_{2}\right) .
\end{array}\right.
$$

Take any $\varepsilon>0$, we are going to show that there is $\lambda \in \Lambda$ such that $T-P_{\lambda} T$ can be uniquely extended to a bounded operator from $L_{u, \eta_{1}}\left(\Gamma_{0}\right) \times L_{v, \eta_{2}}\left(\Gamma_{1}\right)$ to $L_{w, \eta}\left(\Gamma_{2}\right)$ with

$$
\left\|T-P_{\lambda} T\right\|_{L_{u, \eta_{1}}\left(\Gamma_{0}\right) \times L_{v, \eta_{2}}\left(\Gamma_{1}\right), L_{w, \eta}\left(\Gamma_{2}\right)} \leq \varepsilon .
$$

With this aim, put $\widetilde{A}=\left(L_{\infty}\left(\Gamma_{0}\right), L_{1}\left(\Gamma_{0}\right)\right)$, and $\widehat{E}=\left(L_{\infty}\left(\Gamma_{2}\right), L_{\infty}\left(\Gamma_{2}\right)\right)$. By (4.1), we know that $T \in \mathcal{B}(\widetilde{A} \times \bar{B}, \widehat{E})$. Put $\omega=1-1 / w$ and $s=\omega(1 / u-1 / w)^{-1}$. So $1 / u=1-\omega+\omega / s$. Applying the complex bilinear interpolation theorem $[4$, Theorem 4.4.1], with $\tau=1 / s$ we obtain that $T$ may be uniquely extended to a bounded bilinear operator from $L_{s}\left(\Gamma_{0}\right) \times L_{s^{\prime}}\left(\Gamma_{1}\right)$ to $L_{\infty}\left(\Gamma_{2}\right)$. Here $1 / s+1 / s^{\prime}=1$. Therefore

$$
T \in \mathcal{B}\left(\left(L_{1}\left(\Gamma_{0}\right), L_{s}\left(\Gamma_{0}\right)\right) \times\left(L_{1}\left(\Gamma_{1}\right), L_{s^{\prime}}\left(\Gamma_{1}\right)\right),\left(L_{1}\left(\Gamma_{2}\right), L_{\infty}\left(\Gamma_{2}\right)\right)\right) .
$$


The target couple is again $\bar{E}$, so it satisfies $(\mathfrak{H})$. Moreover,

$T: L_{1}\left(\Gamma_{0}\right) \times L_{1}\left(\Gamma_{1}\right) \longrightarrow L_{1}\left(\Gamma_{2}\right)$ is compact and interpolating by the real method we get $\left(L_{1}\left(\Gamma_{0}\right), L_{s}\left(\Gamma_{0}\right)\right)_{\omega, \eta_{1}}=L_{u, \eta_{1}}\left(\Gamma_{0}\right), \quad\left(L_{1}\left(\Gamma_{1}\right), L_{s^{\prime}}\left(\Gamma_{1}\right)\right)_{\omega, \eta_{2}}=L_{v, \eta_{2}}\left(\Gamma_{1}\right)$, $\left(L_{1}\left(\Gamma_{2}\right), L_{\infty}\left(\Gamma_{2}\right)\right)_{\omega, \eta}=L_{w, \eta}\left(\Gamma_{2}\right)$, which are the same spaces as in (4.2). Now applying Proposition 3.4 with $\omega$ and $\eta_{1}, \eta_{2}, \eta$, we derive (4.3).

Observe that the $\lambda$ given by Proposition 3.4 depends on (3.6), that is, on $\omega$ and on the constants $c_{1}, c_{2}$ of the proof of the proposition, constants which only depend on $T, \bar{E}$ and $C$ in (3.2). Whence, if instead of one point we have any finite subset $\left\{\bar{\theta}_{k}\right\}_{k=1}^{m}$ in the interior of $\Omega$, then we can still proceed as before and select a $\lambda$ which works for all $\bar{\theta}_{k}$ with $1 \leq k \leq m$. This yields that $\bar{E}, T$ and $\Omega$ satisfy the condition $(\mathcal{H})$.

To complete the proof, let $1<p, q, r<\infty$, with $1 / p+1 / q=1+1 / r$. Put $\theta_{1}=1-1 / p, \theta_{2}=1-1 / q$ and $\theta=1-1 / r$. Then $0<\theta_{1}, \theta_{2}, \theta<1$ and $\theta=\theta_{1}+\theta_{2}$. Let again $1 \leq \eta_{1}, \eta_{2}<\infty, 1 \leq \eta \leq \infty$ with $1 / \eta_{1}+1 / \eta_{2}=1+1 / \eta$. According to [13, Theorem 5.3], we have

$$
T \in \mathcal{B}\left(L_{p, \eta_{1}}\left(\Gamma_{0}\right) \times L_{q, \eta_{2}}\left(\Gamma_{1}\right), L_{r, \eta}\left(\Gamma_{2}\right)\right) .
$$

and, by (3.4), we know that

$$
\bar{A}_{\theta_{1}, \eta_{1}}=L_{p, \eta_{1}}\left(\Gamma_{0}\right), \bar{B}_{\theta_{2}, \eta_{2}}=L_{q, \eta_{2}}\left(\Gamma_{1}\right), \bar{E}_{\theta, \eta}=L_{r, \eta}\left(\Gamma_{2}\right) .
$$

Hence, $\bar{\theta}=\left(\theta_{1}, \theta_{2}\right)$ and $\bar{\eta}=\left(\eta_{1}, \eta_{2}, \eta\right)$ satisfy $(2.2)$. This yields that $\bar{\theta}$ belongs to the interior of $\Omega$. Consequently, applying Theorem 3.7 with $\bar{\theta}$ and $1 / \alpha+1 / \beta=$ $1 / \gamma$, we derive that $T: L_{p, \alpha}\left(\Gamma_{0}\right) \times L_{q, \beta}\left(\Gamma_{1}\right) \longrightarrow L_{r, \gamma}\left(\Gamma_{2}\right)$ is compact.

Corollary 4.2. Suppose $1<p, q, r<\infty, 1 / 2<s<\infty$, with $1 / p+1 / q=1+1 / r$ and $1 / s=1 / p+1 / q$. Let $T$ be a bilinear operator satisfying (4.1) and such that $T: L_{1}\left(\Gamma_{0}\right) \times L_{1}\left(\Gamma_{1}\right) \longrightarrow L_{1}\left(\Gamma_{2}\right)$ is compact. Then $T$ may be uniquely extended to a compact bilinear operator from $L_{p}\left(\Gamma_{0}\right) \times L_{q}\left(\Gamma_{1}\right)$ to $L_{r, s}\left(\Gamma_{2}\right)$.

Proof. The result follows by applying Theorem 4.1 with $\alpha=p, \beta=q$ and $\gamma=(1 / \alpha+1 \beta)^{-1}=(1 / p+1 / q)^{-1}=s$.

\section{References}

[1] C. Bennet and R. Sharpley, Interpolation of Operators, Academic Press, Boston, 1988.

[2] Á. Bényi and T. Oh, Smoothing of commutators for a Hörmander class of bilinear pseudodifferential operators, J. Fourier Anal. Appl. 20 (2014) 282-300.

[3] Á. Bényi and R.H. Torres, Compact bilinear operators and commutators, Proc. Amer. Math. Soc. 141 (2013) 3609-3621.

[4] J. Bergh and J. Löfström, Interpolation Spaces. An Introduction, Springer, Berlin, 1976.

[5] B.F. Besoy and F. Cobos, Interpolation of the measure of non-compactness of bilinear operators among quasi-Banach spaces, J. Approx. Theory 243 (2019) 25-44. 
[6] A.P. Calderón, Intermediate spaces and interpolation, the complex method, Studia Math. 24 (1964) 113-190.

[7] F. Cobos, L.M. Fernández-Cabrera and A. Martínez, Interpolation of compact bilinear operators among quasi-Banach spaces and applications, Math. Nachr. 291 (2018) 2168-2187.

[8] F. Cobos, L.M. Fernández-Cabrera and A. Martínez, On compactness results of Lions-Peetre type for bilinear operators, Nonlinear Anal. 199 (2020) 111951.

[9] F. Cobos and J. Peetre, Interpolation of compactness using AronszajnGagliardo functors, Israel J. Math. 68 (1989) 220-240.

[10] F. Cobos, T. Kühn and T. Schonbek, One-sided compactness results for Aronszajn-Gagliardo functors, J. Funct. Anal. 106 (1992) 274-313.

[11] D.E. Edmunds and W.D. Evans, Spectral Theory and Differential Operators, Clarendon Press, Oxford, 1987.

[12] L.M. Fernández-Cabrera and A. Martínez, On interpolation properties of compact bilinear operators, Math. Nachr. 290 (2017) 1663-1677.

[13] L.M. Fernández-Cabrera and A. Martínez, Real interpolation of compact bilinear operators, J. Fourier Anal. Appl. 24 (2018) 1181-1203.

[14] D.L. Fernandez and E.B. da Silva, Interpolation of bilinear operators and compactness, Nonlinear Anal. 73 (2010) 526-537.

[15] M.A. Fugarolas and F. Cobos, On Schauder bases in the Lorentz operator ideal, J. Math. Anal. Appl. 95 (1983) 235-242.

[16] I. C. Gohberg and M.G. Krein, Introduction to the Theory of Linear Nonselfadjoint Operators, Amer. Math. Soc., Providence, R.I., 1969.

[17] G. Hu, Compactness of the commutator of bilinear Fourier multiplier operator, Taiwanese J. Math. 18 (2014) 661-675.

[18] R.A. Hunt, On L(p,q) spaces, Enseign. Math. 12 (1966) 249-276.

[19] S. Janson, On interpolation of multilinear operators, in: Function spaces and Applications, Springer Lect. Notes in Math. 1302 (1988) 290-302.

[20] G.E. Karadzhov, The interpolation method of "means" for quasinormed spaces, Doklady Acad. Nauk SSSR 209 (1973) 33-36.

[21] H. König, Interpolation of operator ideals with an application to eigenvalue distribution problems, Math. Ann. 233 (1978) 35-48.

[22] H. König, Eigenvalue distribution of compact operators, Birkhäuser, Basel, 1986.

[23] G. Köthe, Topological Vector Spaces, Vol. I, Springer, New York, 1969.

[24] J.-L. Lions and J. Peetre, Sur une classe d'espaces d'interpolation, Inst. Hautes Études Sci. Publ. Math. 19 (1964) 5-68. 
[25] M. Mastyło and E.B. Silva, Interpolation of the measure of non-compactness of bilinear operators, Trans. Amer. Math. Soc. 370 (2018) 8979-8997.

[26] R. O'Neil, Convolution operators and $L(p, q)$ spaces, Duke Math. J. 30 (1963) 129-142.

[27] A. Persson, Compact linear mappings between interpolation spaces, Ark. Mat. 5 (1964) 215-219.

[28] A. Pietsch, Eigenvalues and s-number, Cambridge University Press, Cambridge, 1987.

[29] M. F. Teixeira and D. E. Edmunds, Interpolation theory and measures of non-compactness, Math. Nachr. 104 (1981) 129-135.

[30] H. Triebel, Über die verteilung der approximationszahlen kompakter operatoren in Sobolev-Besov-Räumen, Invent. Math. 4 (1967) 275-293.

[31] H. Triebel, Interpolation Theory, Function Spaces, Differential Operators, North-Holland, Amsterdam, 1978.

[32] H. Triebel, Theory of Function Spaces III, Birkhäuser, Basel, 2006.

[33] H. Triebel, Function Spaces and Wavelets on Domains, European Math. Soc. Publishing House, Zürich, 2008. 